\title{
Physics of Stars and Measurement Data: Part II
}

\author{
Boris V.Vasiliev \\ Independent Researcher, Russia \\ ${ }^{*}$ Corresponding Author: bv.vasiliev@narod.ru
}

Copyright (c)2014 Horizon Research Publishing All rights reserved.

\begin{abstract}
The explanation of dependencies of the parameters of the stars and the Sun which was measured by astronomers is considered as a main task of the physics of stars. This theory is based on taking into account of the existence of a gravityinduced electric polarization of intra-stellar plasma because this plasma is an electrically polarized substance. The accounting of the gravity-induced electric polarization gives the explanation to data of astronomical measurements: the temperatureradius-mass-luminosity relations, the spectra of seismic oscillations of the Sun, distribution of stars on their masses, magnetic fields of stars and etc. The stellar radiuses, masses and temperatures are expressed by the corresponding ratios of the fundamental constants, and individuality of stars are determined by two parameters - by the charge and mass numbers of nuclei, from which a stellar plasma is composed. This theory is the lack of a collapse in the final stage of the star development, as well as "black holes" that could be results from a such collapse.
\end{abstract}

Keywords Electric Polarization, Plasma, Stellar Mass, Stellar Temperature, Stellar Radius, Seismic Oscillations, Magnetic Field

\section{The thermodynamic relations of intra-stellar plasma}

\subsection{The thermodynamic relation of star atmosphere parameters}

Hot stars steadily generate energy and radiate it from their surfaces. This is non-equilibrium radiation in relation to a star. But it may be a stationary radiation for a star in steady state. Under this condition, the star substance can be considered as an equilibrium. This condition can be considered as quasi-adiabatic, because the interchange of energy between both subsystems - radiation and substance - is stationary and it does not result in a change of entropy of substance. Therefore at consideration of state of a star atmosphere, one can base it on equilibrium conditions of hot plasma and the ideal gas law for adiabatic condition can be used for it in the first approximation.

It is known, that thermodynamics can help to establish correlation between steady-state parameters of a system. Usually, the thermodynamics considers systems at an equilibrium state with constant temperature, constant particle density and constant pressure over all system. The characteristic feature of the considered system is the existence of equilibrium at the absence of a constant temperature and particle density over atmosphere of a star. To solve this problem, one can introduce averaged pressure

$$
\widehat{P} \approx \frac{G \mathbb{M}^{2}}{\mathbb{R}_{0}^{4}},
$$

averaged temperature

$$
\widehat{T}=\frac{\int_{\mathbb{V}} T d V}{V} \sim \mathbb{T}_{0}\left(\frac{\mathbb{R}_{0}}{\mathbb{R}_{\star}}\right)
$$

and averaged particle density

$$
\widehat{n} \approx \frac{\mathbb{N}_{A}}{\mathbb{R}_{0}^{3}}
$$

After it by means of thermodynamical methods, one can find relation between parameters of a star.

\subsubsection{The $c_{P} / c_{V}$ ratio}

At a movement of particles according to the theorem of the equidistribution, the energy $k T / 2$ falls at each degree of freedom. It gives the heat capacity $c_{v}=3 / 2 k$. 
According to the virial theorem $[2,1]$, the full energy of a star should be equal to its kinetic energy (with opposite sign)(see Eq.(I-60)), so as full energy related to one particle

$$
\mathcal{E}=-\frac{3}{2} k T
$$

In this case the heat capacity at constant volume (per particle over Boltzman's constant $k$ ) by definition is

$$
c_{V}=\left(\frac{d E}{d T}\right)_{V}=-\frac{3}{2}
$$

The negative heat capacity of stellar substance is not surprising. It is a known fact and it is discussed in Landau-Lifshitz course [2]. The own heat capacity of each particle of star substance is positive. One obtains the negative heat capacity at taking into account the gravitational interaction between particles.

By definition the heat capacity of an ideal gas particle at permanent pressure [2] is

$$
c_{P}=\left(\frac{d W}{d T}\right)_{P},
$$

where $W$ is enthalpy of a gas.

As for the ideal gas [2]

$$
W-\mathcal{E}=N k T
$$

and the difference between $c_{P}$ and $c_{V}$

$$
c_{P}-c_{V}=1
$$

Thus in the case considered, we have

$$
c_{P}=-\frac{1}{2} .
$$

Supposing that conditions are close to adiabatic ones, we can use the equation of the Poisson's adiabat.

\subsubsection{The Poisson's adiabat}

The thermodynamical potential of a system consisting of $N$ molecules of ideal gas at temperature $T$ and pressure $P$ can be written as [2]:

$$
\Phi=\text { const } \cdot N+N T \ln P-N c_{P} T \ln T
$$

The entropy of this system

$$
S=\text { const } \cdot N-N \ln P+N c_{P} \ln T \text {. }
$$

As at adiabatic process, the entropy remains constant

$$
-N T \ln P+N c_{P} T \ln T=\text { const, }
$$

we can write the equation for relation of averaged pressure in a system with its volume (The Poisson's adiabat) [2]:

$$
\widehat{P} V^{\widetilde{\gamma}}=\text { const }
$$

where $\widetilde{\gamma}=\frac{c_{P}}{c_{V}}$ is the exponent of adiabatic constant. In considered case taking into account of Eqs.(6) and (5), we obtain

$$
\widetilde{\gamma}=\frac{c_{P}}{c_{V}}=\frac{1}{3} .
$$

As $V^{1 / 3} \sim \mathbb{R}_{0}$, we have for equilibrium condition

$$
\widehat{P} \mathbb{R}_{0}=\text { const. }
$$

\subsection{The mass-radius ratio}

As it was shown in Part I, there is energetically favorable state of dense plasma. The equilibrium plasma density in this state is defined by Eq. (I-22) and its equilibrium temperature is defined by Eq.(I-26). Based on this in Part I the expression for the equilibrium value of the stellar mass Eq.(1-57) was obtained .

$$
\frac{\mathbb{M}^{2}}{\mathbb{R}_{0}^{3}}=\text { const }
$$

This equation shows the existence of internal constraint of chemical parameters of equilibrium state of a star. Indeed, the substitution of obtained determinations Eq.(I-94) and Eq.(I-95) into Eq.(16) gives:

$$
Z \sim(A / Z)^{5 / 6}
$$




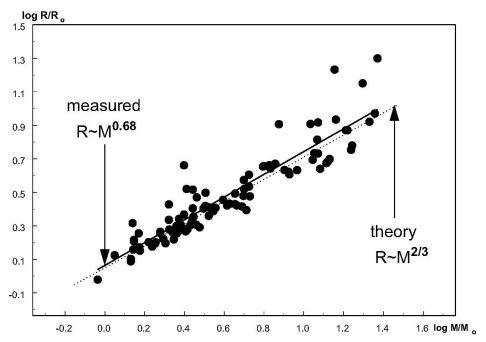

Figure 1. The dependence of radii of stars over the star mass [3]. Here the radius of stars is normalized to the sunny radius, the stars masses are normalized to the mass of the Sum. The data are shown on double logarithmic scale. The solid line shows the result of fitting of measurement data $\mathbb{R}_{0} \sim \mathbb{M}^{0.68}$. The theoretical dependence $\mathbb{R}_{0} \sim \mathbb{M}^{2 / 3}(16)$ is shown by the dotted line.

Simultaneously the observational data of masses, of radii and their temperatures was obtained by astronomers for close binary stars [3]. The dependence of radii of these stars over these masses is shown in Fig.1 on double logarithmic scale. The solid line shows the result of fitting of measurement data $\mathbb{R}_{0} \sim \mathbb{M}^{0.68}$. It is close to theoretical dependence $\mathbb{R}_{0} \sim \mathbb{M}^{2 / 3}($ Eq.16) which is shown by dotted line.

If parameters of the star are expressed through corresponding solar values $\rho \equiv \frac{\mathbb{R}_{0}}{\mathbb{R}_{\odot}}$ and $\mu \equiv \frac{\mathbb{M}}{\mathbb{M}_{\odot}}$, that Eq.(16) can be rewritten as

$$
\frac{\rho}{\mu^{2 / 3}}=1
$$

Numerical values of relations $\frac{\rho}{\mu^{2 / 3}}$ for close binary stars [3] are shown in the Table 1 .

\begin{tabular}{|c|c|c|c|c|c|c|c|c|}
\hline $\mathrm{N}$ & Star & & $\mu \equiv \frac{\mathbb{M}}{\mathbb{M} \odot}$ & $\rho \equiv \frac{\mathbb{R}_{0}}{\mathbb{R}_{\odot}}$ & $\tau \equiv \frac{\mathbb{T}_{0}}{\mathbb{T}_{\odot}}$ & $\frac{\rho}{\mu^{2 / 3}}$ & $\frac{\tau}{\mu^{7 / 12}}$ & $\frac{\rho \tau}{\mu^{5 / 4}}$ \\
\hline \multirow{2}{*}{1} & \multirow{2}{*}{ BW Aqr } & 1 & 1.48 & 1.803 & 1.043 & 1.38 & 0.83 & 1.15 \\
\hline & & 2 & 1.38 & 2.075 & 1.026 & 1.67 & 0.85 & 1.42 \\
\hline \multirow{2}{*}{2} & \multirow{2}{*}{ V $889 \mathrm{Aql}$} & 1 & 2.4 & 2.028 & 1.692 & 1.13 & 1.01 & 1.15 \\
\hline & & 2 & 2.2 & 1.826 & 1.607 & 1.08 & 1.01 & 1.09 \\
\hline \multirow{2}{*}{3} & \multirow{2}{*}{ V 539 Ara } & 1 & 6.24 & 4.512 & 3.043 & 1.33 & 1.04 & 1.39 \\
\hline & & 2 & 5.31 & 4.512 & 3.043 & 1.12 & 1.09 & 1.23 \\
\hline \multirow{2}{*}{4} & \multirow{2}{*}{ AS Cam } & 1 & 3.31 & 2.58 & 1.966 & 1.16 & 0.98 & 1.13 \\
\hline & & 2 & 2.51 & 1.912 & 1.709 & 1.03 & 1.0 & 1.03 \\
\hline \multirow{2}{*}{5} & \multirow{2}{*}{ EM Car } & 1 & 22.8 & 9.35 & 5.658 & 1.16 & 0.91 & 1.06 \\
\hline & & 2 & 21.4 & 8.348 & 5.538 & 1.08 & 0.93 & 1.00 \\
\hline \multirow{2}{*}{6} & \multirow{2}{*}{ GL Car } & 1 & 13.5 & 4.998 & 5.538 & 0.88 & 1.08 & 0.95 \\
\hline & & 2 & 13 & 4.726 & 4.923 & 0.85 & 1.1 & 0.94 \\
\hline \multirow{2}{*}{7} & \multirow{2}{*}{ QX Car } & 1 & 9.27 & 4.292 & 4 & 0.97 & 1.09 & 1.06 \\
\hline & & 2 & 8.48 & 4.054 & 3.829 & 0.975 & 1.1 & 1.07 \\
\hline \multirow{2}{*}{8} & \multirow{2}{*}{ AR Cas } & 1 & 6.7 & 4.591 & 3.111 & 1.29 & 1.02 & 1.32 \\
\hline & & 2 & 1.9 & 1.808 & 1.487 & 1.18 & 1.02 & 1.21 \\
\hline \multirow{2}{*}{9} & \multirow{2}{*}{ IT Cas } & 1 & 1.4 & 1.616 & 1.102 & 1.29 & 0.91 & 1.17 \\
\hline & & 2 & 1.4 & 1.644 & 1.094 & 1.31 & 0.90 & 1.18 \\
\hline \multirow{2}{*}{10} & \multirow{2}{*}{ OX Cas } & 1 & 7.2 & 4.69 & 4.068 & 1.25 & 1.29 & 1.62 \\
\hline & & 2 & 6.3 & 4.54 & 3.93 & 1.33 & 1.34 & 1.79 \\
\hline \multirow{2}{*}{11} & \multirow{2}{*}{ PV Cas } & 1 & 2.79 & 2.264 & 1.914 & 1.14 & 1.05 & 1.20 \\
\hline & & 2 & 2.79 & 2.264 & 2.769 & 1.14 & 1.05 & 1.20 \\
\hline \multirow{2}{*}{12} & \multirow{2}{*}{ KT Cen } & 1 & 5.3 & 4.028 & 2.769 & 1.32 & 1.05 & 1.39 \\
\hline & & 2 & 5 & 3.745 & 2.701 & 1.28 & 1.06 & 1.35 \\
\hline
\end{tabular}

Table 1. The relations of main stellar parameters 


\begin{tabular}{|c|c|c|c|c|c|c|c|c|}
\hline $\mathrm{N}$ & Star & $\mathrm{n}$ & $\mu \equiv \frac{\mathbb{M}}{\mathbb{M}_{\odot}}$ & $\rho \equiv \frac{\mathbb{R}_{0}}{\mathbb{R}_{\odot}}$ & $\tau \equiv \frac{\mathbb{T}_{0}}{\mathbb{T}_{\odot}}$ & $\frac{\rho}{\mu^{2 / 3}}$ & $\frac{\tau}{\mu^{7 / 12}}$ & $\frac{\rho \tau}{\mu^{5 / 4}}$ \\
\hline \multirow{2}{*}{13} & \multirow{2}{*}{ V 346 Cen } & 1 & 11.8 & 8.26 & 4.05 & 1.59 & 0.96 & 1.53 \\
\hline & & 2 & 8.4 & 4.19 & 3.83 & 1.01 & 1.11 & 1.12 \\
\hline \multirow{2}{*}{14} & \multirow{2}{*}{ CW Cep } & 1 & 11.8 & 8.263 & 4.051 & 1.04 & 1.06 & 1.11 \\
\hline & & 2 & 11.1 & 4.954 & 4.393 & 1.0 & 1.08 & 1.07 \\
\hline \multirow{2}{*}{15} & \multirow{2}{*}{ EK Cep } & 1 & 2.02 & 1.574 & 1.709 & 0.98 & 1.13 & 1.12 \\
\hline & & 2 & 1.12 & 1.332 & 1.094 & 1.23 & 1.02 & 1.26 \\
\hline \multirow{2}{*}{16} & \multirow{2}{*}{$\alpha \mathrm{Cr} \mathrm{B}$} & 1 & 2.58 & 3.314 & 1.555 & 1.76 & 0.89 & 1.57 \\
\hline & & 2 & 0.92 & 0.955 & 0.923 & 1.01 & 0.97 & 0.98 \\
\hline \multirow{2}{*}{17} & \multirow{2}{*}{ Y Cyg } & 1 & 17.5 & 6.022 & 5.66 & 0.89 & 1.06 & 0.95 \\
\hline & & 2 & 17.3 & 5.68 & 5.54 & 0.85 & 1.05 & 0.89 \\
\hline \multirow{2}{*}{18} & \multirow{2}{*}{ Y 380 Cyg } & 1 & 14.3 & 17.08 & 3.54 & 2.89 & 0.75 & 2.17 \\
\hline & & 2 & 8 & 4.3 & 3.69 & 1.07 & 1.1 & 1.18 \\
\hline \multirow{2}{*}{19} & \multirow{2}{*}{ V 453 Cyg } & 1 & 14.5 & 8.607 & 4.55 & 1.45 & 0.95 & 1.38 \\
\hline & & 2 & 11.3 & 5.41 & 4.44 & 1.07 & 1.08 & 1.16 \\
\hline \multirow{2}{*}{20} & \multirow{2}{*}{ V 477 Cyg } & 1 & 1.79 & 1.567 & 1.46 & 1.06 & 1.04 & 1.11 \\
\hline & & 2 & 1.35 & 1.27 & 1.11 & 1.04 & 0.93 & 0.97 \\
\hline \multirow{2}{*}{21} & \multirow{2}{*}{ V 478 Cyg } & 1 & 16.3 & 7.42 & 5.09 & 1.15 & 1.0 & 1.15 \\
\hline & & 2 & 16.6 & 7.42 & 5.09 & 1.14 & 0.99 & 1.13 \\
\hline \multirow{2}{*}{22} & \multirow{2}{*}{ V 541 Cyg } & 1 & 2.69 & 2.013 & 1.86 & 1.04 & 1.05 & 1.09 \\
\hline & & 2 & 2.6 & 1.9 & 1.85 & 1.0 & 1.6 & 1.06 \\
\hline \multirow{2}{*}{23} & \multirow{2}{*}{ V 1143 Cyg } & 1 & 1.39 & 1.44 & 1.11 & 1.16 & 0.92 & 0.92 \\
\hline & & 2 & 1.35 & 1.23 & 1.09 & 1.0 & 0.91 & 0.92 \\
\hline \multirow{2}{*}{24} & \multirow{2}{*}{ V 1765 Cyg } & 1 & 23.5 & 19.96 & 4.39 & 2.43 & 0.67 & 1.69 \\
\hline & & 2 & 11.7 & 6.52 & 4.29 & 1.26 & 1.02 & 1.29 \\
\hline
\end{tabular}

The Table 1(continuation). 
The Table 1(continuation).

\begin{tabular}{|c|c|c|c|c|c|c|c|c|}
\hline $\mathrm{N}$ & Star & $\mathrm{n}$ & $\mu \equiv \frac{\mathbb{M}}{\mathbb{M} \odot}$ & $\rho \equiv \frac{\mathbb{R}_{0}}{\mathbb{R}_{\odot}}$ & $\tau \equiv \frac{\mathbb{T}_{0}}{\mathbb{T}_{\odot}}$ & $\frac{\rho}{\mu^{2 / 3}}$ & $\frac{\tau}{\mu^{7 / 12}}$ & $\frac{\rho \tau}{\mu^{5 / 4}}$ \\
\hline \multirow{2}{*}{25} & \multirow{2}{*}{ DI Her } & 1 & 5.15 & 2.48 & 2.91 & 0.83 & 1.12 & 0.93 \\
\hline & & 2 & 4.52 & 2.69 & 2.58 & 0.98 & 1.07 & 1.05 \\
\hline \multirow{2}{*}{26} & \multirow{2}{*}{ HS Her } & 1 & 4.25 & 2.71 & 2.61 & 1.03 & 1.12 & 1.16 \\
\hline & & 2 & 1.49 & 1.48 & 1.32 & 1.14 & 1.04 & 1.19 \\
\hline \multirow{2}{*}{27} & \multirow{2}{*}{ CO Lac } & 1 & 3.13 & 2.53 & 1.95 & 1.18 & 1.00 & 1.12 \\
\hline & & 2 & 2.75 & 2.13 & 1.86 & 1.08 & 1.01 & 1.09 \\
\hline \multirow{2}{*}{28} & \multirow{2}{*}{ GG Lup } & 1 & 6.24 & 4.12 & 2.64 & 1.03 & 1.08 & 1.11 \\
\hline & & 2 & 2.51 & 1.92 & 1.79 & 1.04 & 1.05 & 1.09 \\
\hline \multirow{2}{*}{29} & \multirow{2}{*}{ RU Mon } & 1 & 3.6 & 2.55 & 2.20 & 1.09 & 1.04 & 1.14 \\
\hline & & 2 & 3.33 & 2.29 & 2.15 & 1.03 & 1.07 & 1.10 \\
\hline \multirow{2}{*}{30} & \multirow{2}{*}{ GN Nor } & 1 & 2.5 & 4.59 & 1.33 & 2.49 & 0.78 & 1.95 \\
\hline & & 2 & 2.5 & 4.59 & 1.33 & 2.49 & 0.78 & 1.95 \\
\hline \multirow{2}{*}{31} & \multirow{2}{*}{ U Oph } & 1 & 5.02 & 3.31 & 2.80 & 1.13 & 1.09 & 1.23 \\
\hline & & 2 & 4.52 & 3.11 & 2.60 & 1.14 & 1.08 & 1.23 \\
\hline \multirow{2}{*}{32} & \multirow{2}{*}{ V $451 \mathrm{Oph}$} & 1 & 2.77 & 2.54 & 1.86 & 1.29 & 1.03 & 1.32 \\
\hline & & 2 & 2.35 & 1.86 & 1.67 & 1.05 & 1.02 & 1.07 \\
\hline \multirow{2}{*}{33} & \multirow{2}{*}{$\beta$ Ori } & 1 & 19.8 & 14.16 & 4.55 & 1.93 & 0.80 & 1.54 \\
\hline & & 2 & 7.5 & 8.07 & 3.04 & 2.11 & 0.94 & 1.98 \\
\hline \multirow{2}{*}{34} & \multirow{2}{*}{ FT Ori } & 1 & 2.5 & 1.89 & 1.81 & 1.03 & 1.06 & 1.09 \\
\hline & & 2 & 2.3 & 1.80 & 1.62 & 1.03 & 1.0 & 1.03 \\
\hline \multirow{2}{*}{35} & \multirow{2}{*}{ AG Per } & 1 & 5.36 & 3.0 & 2.91 & 0.98 & 1.09 & 1.06 \\
\hline & & 2 & 4.9 & 2.61 & 2.91 & 0.90 & 1.15 & 1.04 \\
\hline \multirow{2}{*}{36} & \multirow{2}{*}{ IQ Per } & 1 & 3.51 & 2.44 & 2.27 & 1.06 & 1.09 & 1.16 \\
\hline & & 2 & 1.73 & 1.50 & 2.27 & 1.04 & 1.00 & 1.05 \\
\hline
\end{tabular}




\begin{tabular}{|c|c|c|c|c|c|c|c|c|}
\hline $\mathrm{N}$ & Star & $\mathrm{n}$ & $\mu \equiv \frac{\mathbb{M}}{\mathbb{M} \odot}$ & $\rho \equiv \frac{\mathbb{R}_{0}}{\mathbb{R}_{\odot}}$ & $\tau \equiv \frac{\mathbb{T}_{0}}{\mathbb{T}_{\odot}}$ & $\frac{\rho}{\mu^{2 / 3}}$ & $\frac{\tau}{\mu^{7 / 12}}$ & $\frac{\rho \tau}{\mu^{5 / 4}}$ \\
\hline \multirow{2}{*}{37} & \multirow{2}{*}{$\varsigma$ Phe } & 1 & 3.93 & 2.85 & 2.41 & 1.14 & 1.08 & 1.24 \\
\hline & & 2 & 2.55 & 1.85 & 1.79 & 0.99 & 1.04 & 1.03 \\
\hline \multirow{2}{*}{38} & \multirow{2}{*}{ KX Pup } & 1 & 2.5 & 2.33 & 1.74 & 1.27 & 1.02 & 1.29 \\
\hline & & 2 & 1.8 & 1.59 & 1.38 & 1.08 & 0.98 & 1.06 \\
\hline \multirow{2}{*}{39} & \multirow{2}{*}{ NO Pup } & 1 & 2.88 & 2.03 & 1.95 & 1.00 & 1.05 & 1.05 \\
\hline & & 2 & 1.5 & 1.42 & 1.20 & 1.08 & 0.94 & 1.02 \\
\hline \multirow{2}{*}{40} & \multirow{2}{*}{ VV Pyx } & 1 & 2.1 & 2.17 & 1.49 & 1.32 & 0.96 & 1.27 \\
\hline & & 2 & 2.1 & 2.17 & 1.49 & 1.32 & 0.96 & 1.27 \\
\hline \multirow{2}{*}{41} & \multirow{2}{*}{ YY Sgr } & 1 & 2.36 & 2.20 & 1.59 & 1.24 & 0.96 & 1.19 \\
\hline & & 2 & 2.29 & 1.99 & 1.59 & 1.15 & 0.98 & 1.12 \\
\hline \multirow{2}{*}{42} & \multirow{2}{*}{ V $523 \mathrm{Sgr}$} & 1 & 2.1 & 2.67 & 1.42 & 1.63 & 0.92 & 1.50 \\
\hline & & 2 & 1.9 & 1.84 & 1.42 & 1.20 & 0.98 & 1.17 \\
\hline \multirow{2}{*}{43} & \multirow{2}{*}{ V $526 \mathrm{Sgr}$} & 1 & 2.11 & 1.9 & 1.30 & 1.15 & 0.84 & 0.97 \\
\hline & & 2 & 1.66 & 1.60 & 1.30 & 1.14 & 0.97 & 1.10 \\
\hline \multirow{2}{*}{44} & \multirow{2}{*}{ V 1647 Sgr } & 1 & 2.19 & 1.83 & 1.52 & 1.09 & 0.96 & 1.05 \\
\hline & & 2 & 1.97 & 1.67 & 4.44 & 1.06 & 1.02 & 1.09 \\
\hline \multirow{2}{*}{45} & \multirow{2}{*}{ V $2283 \mathrm{Sgr}$} & 1 & 3.0 & 1.96 & 1.67 & 0.94 & 0.88 & 0.83 \\
\hline & & 2 & 2.22 & 1.66 & 1.67 & 0.97 & 1.05 & 1.02 \\
\hline \multirow{2}{*}{46} & \multirow{2}{*}{ V 760 Sco } & 1 & 4.98 & 3.02 & 2.70 & 1.03 & 1.06 & 1.09 \\
\hline & & 2 & 4.62 & 2.64 & 2.70 & 0.95 & 1.11 & 1.05 \\
\hline \multirow{2}{*}{47} & \multirow{2}{*}{ AO Vel } & 1 & 3.2 & 2.62 & 1.83 & 1.21 & 0.93 & 1.12 \\
\hline & & 2 & 2.9 & 2.95 & 1.83 & 1.45 & 0.98 & 1.43 \\
\hline \multirow{2}{*}{48} & \multirow{2}{*}{ EO Vel } & 1 & 3.21 & 3.14 & 1.73 & 1.44 & 0.87 & 1.26 \\
\hline & & 2 & 2.77 & 3.28 & 1.73 & 1.66 & 0.95 & 1.58 \\
\hline \multirow{2}{*}{49} & \multirow{2}{*}{$\alpha$ Vir } & 1 & 10.8 & 6.10 & 3.25 & 1.66 & 0.81 & 1.34 \\
\hline & & 2 & 6.8 & 4.39 & 3.25 & 1.22 & 1.06 & 1.30 \\
\hline \multirow{2}{*}{50} & \multirow{2}{*}{ DR Vul } & 1 & 13.2 & 4.81 & 4.79 & 0.83 & 1.06 & 0.91 \\
\hline & & 2 & 12.1 & 4.37 & 4.79 & 0.83 & 1.12 & 0.93 \\
\hline
\end{tabular}

The Table 1(continuation).

\subsection{The mass-temperature and mass-luminosity relations}

As there are the expressions for energetically favorable temperature of the star core Eq.(I-26) and for core's radius Eq.(I45), with using the dependence Eq.(I-55), one can obtain the relation between surface temperature and the radius of a star

$$
\mathbb{T}_{0} \sim \mathbb{R}_{0}^{7 / 8}
$$

or accounting for (16)

$$
\mathbb{T}_{0} \sim \mathbb{M}^{7 / 12}
$$

The dependence of the temperature on the star surface over the star mass of close binary stars [3] is shown in Fig.(2). Here the temperatures of stars are normalized to the sunny surface temperature $(5875 \mathrm{C})$, the stars masses are normalized to the mass of the Sum. The data are shown on double logarithmic scale. The solid line shows the result of fitting of measurement data $\left(\mathbb{T}_{0} \sim \mathbb{M}^{0.59}\right)$. The theoretical dependence $\mathbb{T}_{0} \sim \mathbb{M}^{7 / 12}$ (Eq.20) is shown by dotted line.

If parameters of the star are expressed through corresponding solar values $\tau \equiv \frac{\mathbb{T}_{0}}{\mathbb{T}_{\odot}}$ and $\mu \equiv \frac{\mathbb{M}}{\mathbb{M} \odot}$, that Eq.(20) can be rewritten as

$$
\frac{\tau}{\mu^{7 / 12}}=1
$$


Numerical values of relations $\frac{\tau}{\mu^{7 / 12}}$ for close binary stars [3] are shown in the Table 1.

The analysis of these data leads to few conclusions. The averaging over all tabulated stars gives

$$
<\frac{\tau}{\mu^{7 / 12}}>=1.007 \pm 0.07
$$

and we can conclude that the variability of measured data of surface temperatures and stellar masses has statistical character. Secondly, Eq.(21) is valid for all hot stars (exactly for all stars which are gathered in Tab. 1).

The problem with the averaging of $\frac{\rho}{\mu^{2 / 3}}$ looks different. There are a few of giants and super-giants in this Table. The values of ratio $\frac{\rho}{\mu^{2 / 3}}$ are more than 2 for them. It seems that, if to exclude these stars from consideration, the averaging over stars of the main sequence gives value close to 1. Evidently, it needs in more detail consideration.

The luminosity of a star

$$
\mathbb{L}_{0} \sim \mathbb{R}_{0}^{2} \mathbb{T}_{0}^{4}
$$

at taking into account (Eq.16) and (Eq.20) can be expressed as

$$
\mathbb{L}_{0} \sim \mathbb{M}^{11 / 3} \sim \mathbb{M}^{3.67}
$$

This dependence is shown in Fig.(3) It can be seen that all calculated interdependencies $\mathbb{R}(\mathbb{M}), \mathbb{T}(\mathbb{M})$ and $L(\mathbb{M})$ show a good qualitative agreement with the measuring data. At that it is important, that the quantitative explanation of mass-luminosity dependence discovered at the beginning of 20 th century is obtained.

\subsubsection{The compilation of the results of calculations}

Let us put together the results of calculations. It is energetically favorable for the star to be divided into two volumes: the core is located in the central area of the star and the atmosphere is surrounding it from the outside. (Fig.4). The core has the radius:

$$
\mathbb{R}_{\star}=2.08 \frac{a_{B}}{Z(A / Z)}\left(\frac{\hbar c}{G m_{p}^{2}}\right)^{1 / 2} \approx \frac{1.41 \cdot 10^{11}}{Z(A / Z)} \mathrm{cm} .
$$

It is roughly equal to $1 / 10$ of the stellar radius.

At that the mass of the core is equal to

$$
\mathbb{M}_{\star}=6.84 \frac{\mathbb{M}_{C h}}{\left(\frac{A}{Z}\right)^{2}} .
$$

It is almost exactly equal to one half of the full mass of the star.

The plasma inside the core has the constant density

$$
n_{\star}=\frac{16}{9 \pi} \frac{Z^{3}}{a_{B}^{3}} \approx 1.2 \cdot 10^{24} Z^{3} \mathrm{~cm}^{-3}
$$

and constant temperature

$$
\mathbb{T}_{\star}=\left(\frac{25 \cdot 13}{28 \pi^{4}}\right)^{1 / 3}\left(\frac{\hbar c}{k a_{B}}\right) Z \approx Z \cdot 2.13 \cdot 10^{7} K .
$$

The plasma density and its temperature are decreasing at an approaching to the stellar surface:

$$
n_{e}(r)=n_{\star}\left(\frac{\mathbb{R}_{\star}}{r}\right)^{6}
$$

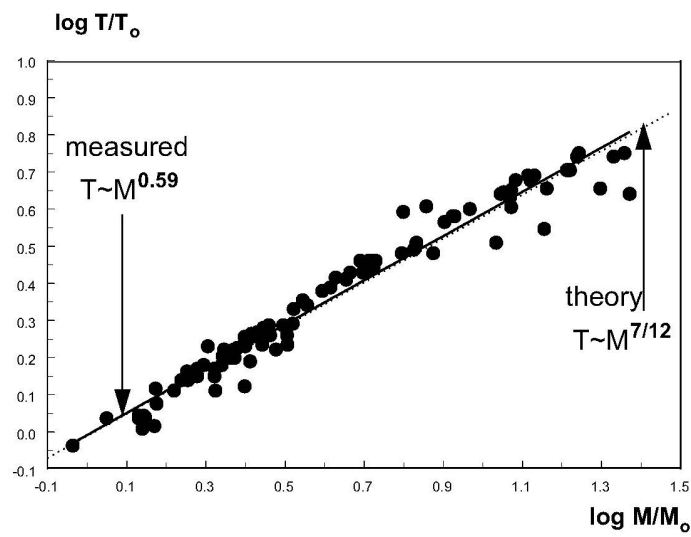

Figure 2. The dependence of the temperature on the star surface over the star mass of close binary stars [3]. Here the temperatures of stars are normalized to surface temperature of the Sun $(5875 \mathrm{C})$, the stars masses are normalized to the mass of Sum. The data are shown on double logarithmic scale. The solid line shows the result of fitting of measurement data $\left(\mathbb{T}_{0} \sim \mathbb{M}^{0.59}\right)$. The theoretical dependence $\mathbb{T}_{0} \sim \mathbb{M}^{7 / 12}($ Eq.20) is shown by dotted line. 


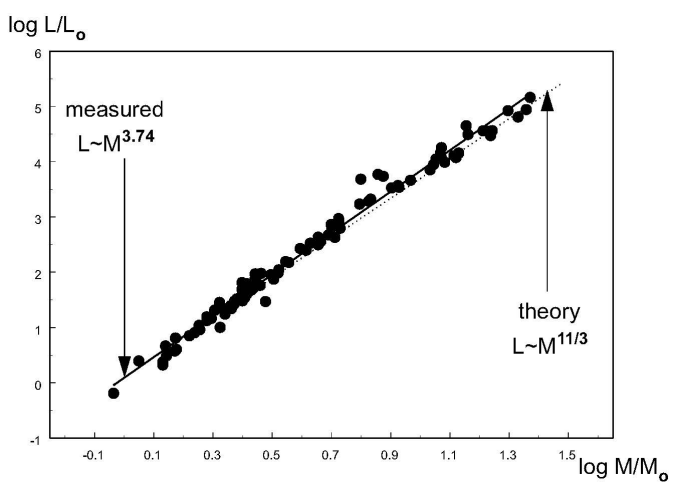

Figure 3. The dependence of star luminosity on the star mass of close binary stars [3]. The luminosities are normalized to the luminosity of the Sun, the stars masses are normalized to the mass of the Sum. The data are shown on double logarithmic scale. The solid line shows the result of fitting of measurement data $\mathbb{L} \sim \mathbb{M}^{3.74}$. The theoretical dependence $\mathbb{L} \sim \mathbb{M}^{11 / 3}$ (Eq.24) is shown by dotted line.

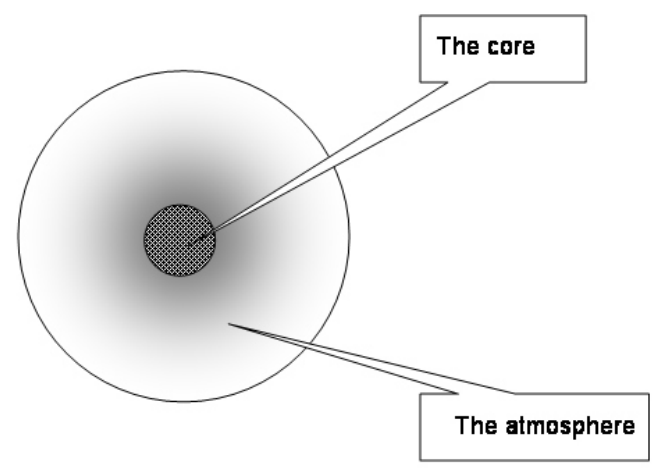

Figure 4. The schematic of the star interior

and

$$
T_{r}=\mathbb{T}_{\star}\left(\frac{\mathbb{R}_{\star}}{r}\right)^{4}
$$

The external radius of the star is determined as

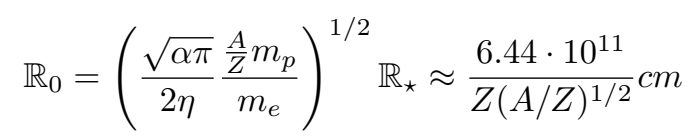

and the temperature on the stellar surface is equal to

$$
\mathbb{T}_{0}=\mathbb{T}_{\star}\left(\frac{\mathbb{R}_{\star}}{\mathbb{R}_{0}}\right)^{4} \approx 4.92 \cdot 10^{5} \frac{Z}{(A / Z)^{2}}
$$

\section{Magnetic fields and magnetic moments of stars}

\subsection{Magnetic moments of celestial bodies}

A thin spherical surface with radius $r$ carrying an electric charge $q$ at the rotation around its axis with frequency $\Omega$ obtains the magnetic moment

$$
\mathfrak{m}=\frac{r^{2}}{3 c} q \boldsymbol{\Omega} .
$$

The rotation of a ball charged at density $\varrho(r)$ will induce the magnetic moment

$$
\boldsymbol{\mu}=\frac{\boldsymbol{\Omega}}{3 c} \int_{0}^{R} r^{2} \varrho(r) 4 \pi r^{2} d r .
$$

Thus the positively charged core of a star induces the magnetic moment

$$
\mathfrak{m}_{+}=\frac{\sqrt{G} \mathbb{M}_{\star} \mathbb{R}_{\star}^{2}}{5 c} \boldsymbol{\Omega} .
$$

A negative charge will be concentrated in the star atmosphere. The absolute value of atmospheric charge is equal to the positive charge of a core. As the atmospheric charge is placed near the surface of a star, its magnetic moment will be more 


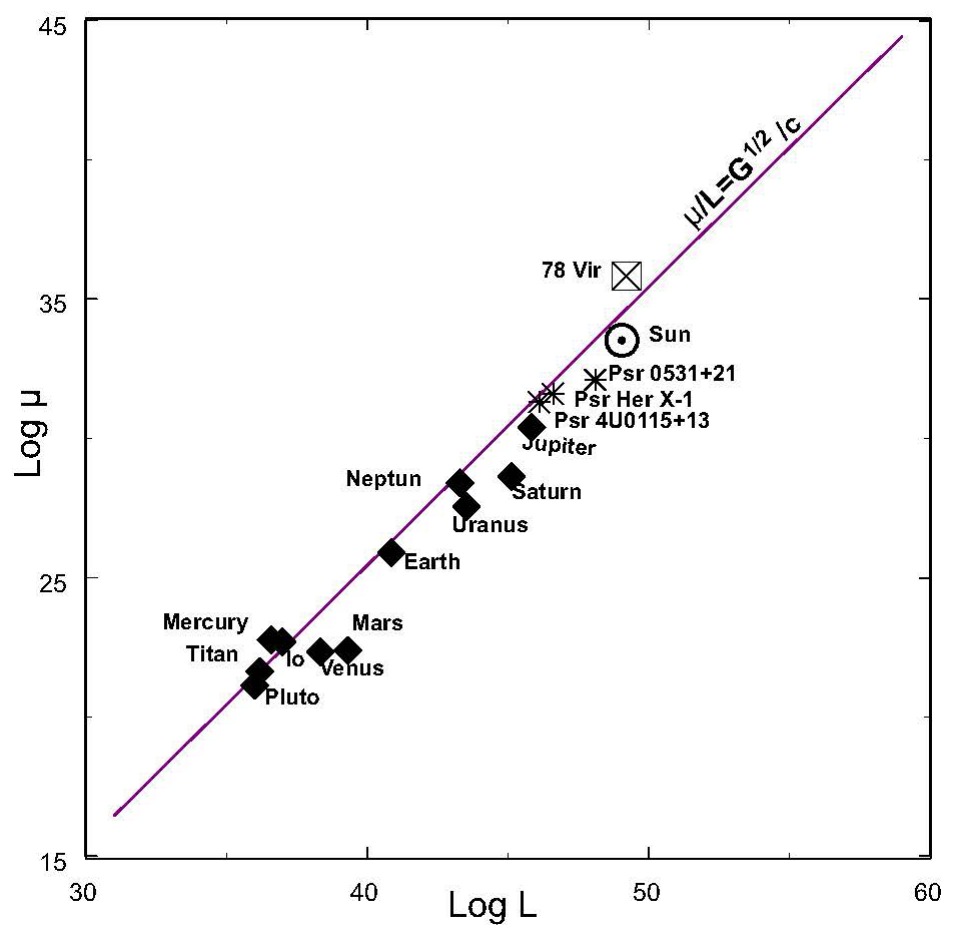

Figure 5. The observed values of magnetic moments of celestial bodies vs. their angular momenta [5]. In ordinate, the logarithm of the magnetic moment (in $\mathrm{Gs} \cdot \mathrm{cm}^{3}$ ) is plotted; in abscissa the logarithm of the angular momentum (in $\mathrm{erg} \cdot \mathrm{s}$ ) is shown. The solid line illustrates Eq.(38). The dash-dotted line fits of observed values.

than the core magnetic moment. The calculation shows that as a result, the total magnetic moment of the star will have the same order of magnitude as the core but it will be negative:

$$
\mathfrak{m}_{\Sigma} \approx-\frac{\sqrt{G}}{c} \mathbb{M}_{\star} \mathbb{R}_{\star}^{2} \boldsymbol{\Omega}
$$

Simultaneously, the torque of a ball with mass $\mathbb{M}$ and radius $\mathbb{R}$ is

$$
\mathcal{L} \approx \mathbb{M}_{\star} \mathbb{R}_{\star}^{2} \Omega .
$$

As a result, for celestial bodies where the force of their gravity induces the electric polarization according to Eq.(I-39), the giromagnetic ratio will depend on world constants only:

$$
\frac{\mathfrak{m}_{\Sigma}}{\mathcal{L}} \approx-\frac{\sqrt{G}}{c}
$$

This relation was obtained for the first time by P.M.S.Blackett [4]. He shows that giromagnetic ratios of the Earth, the Sun and the star 78 Vir are really near to $\sqrt{G} / c$.

By now the magnetic fields, masses, radii and velocities of rotation are known for all planets of the Solar system and for a some stars [5]. These measuring data are shown in Fig.(5), which is taken from [5]. It is possible to see that these data are in satisfactory agreement with Blackett's ratio. At some assumption, the same parameters can be calculated for pulsars. All measured masses of pulsars are equal by the order of magnitude [7]. It is in satisfactory agreement with the condition of equilibrium of relativistic matter (see ). It gives a possibility to consider that masses and radii of pulsars are determined. According to generally accepted point of view, pulsar radiation is related with its rotation, and it gives their rotation velocity. These assumptions permit to calculate the giromagnetic ratios for three pulsars with known magnetic fields on their poles [6]. It is possible to see from Fig.(5), the giromagnetic ratios of these pulsars are in agreement with Blackett's ratio.

\subsection{Magnetic fields of hot stars}

At the estimation of the magnetic field on the star pole, it is necessary to find the field which is induced by stellar atmosphere. The field which is induced by stellar core is small because $\mathbb{R}_{\star} \ll R_{0}$. The field of atmosphere

$$
\mathfrak{m}_{-}=\frac{\boldsymbol{\Omega}}{3 c} \int_{R_{\star}}^{R_{0}} 4 \pi \frac{d i v \mathfrak{P}}{3} r^{4} d r .
$$

can be calculated numerically. But, for our purpose it is enough to estimate this field in order of value:

$$
\mathcal{H} \approx \frac{2 \mathfrak{m}_{-}}{R_{0}^{3}} .
$$




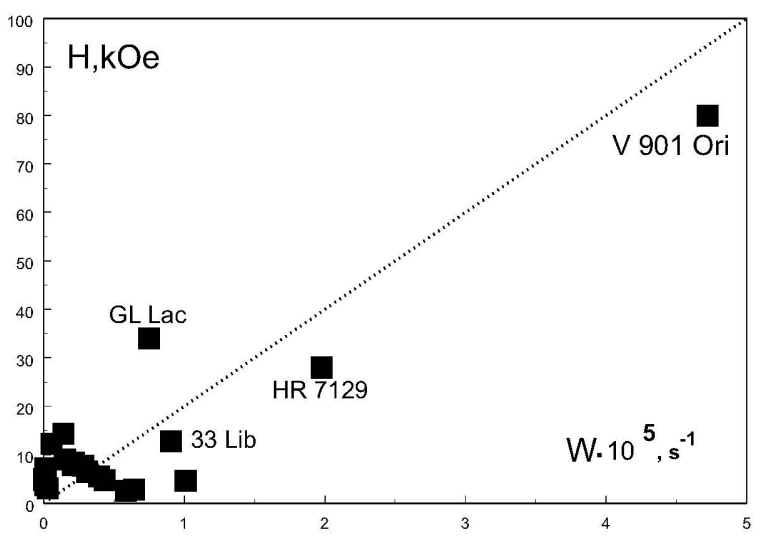

Figure 6. The dependence of magnetic fields on poles of Ap-stars as a function of their rotation velocity [8]. The line shows Eq.(43)).

As

$$
\mathfrak{m}_{-} \approx \frac{\sqrt{G} 2 M_{\star} R_{0}^{2}}{c} \boldsymbol{\Omega}
$$

the field on the star pole

$$
\mathcal{H} \approx-4 \frac{\sqrt{G} \mathbb{M}_{\star}}{c R_{0}} \Omega
$$

At taking into account above obtained relations, one can see that this field is weakly depending on $Z$ and $A / Z$, i.e. on the star temperature, on the star radius and mass. It depends linearly on the velocity of star rotation only:

$$
\mathcal{H} \approx-50\left(\frac{m_{e}}{m_{p}}\right)^{3 / 2} \frac{\alpha^{3 / 4} c}{\sqrt{G}} \boldsymbol{\Omega} \approx-2 \cdot 10^{9} \boldsymbol{\Omega} \quad \text { Oe. }
$$

The magnetic fields are measured for stars of Ap-class [8]. These stars are characterized by changing their brightness in time. The periods of these changes are measured too. At present there is no full understanding of causes of these visible changes of the luminosity. If these luminosity changes caused by some internal reasons will occur not uniformly on a star surface, one can conclude that the measured period of the luminosity change can depend on star rotation. It is possible to think that at relatively rapid rotation of a star, the period of a visible change of the luminosity can be determined by this rotation in general. To check this suggestion, we can compare the calculated dependence (Eq.43) with measuring data [8] (see Fig. 6). Evidently one must not expect very good coincidence of calculations and measuring data, because calculations were made for the case of a spherically symmetric model and measuring data are obtained for stars where this symmetry is obviously violated. So getting consent on order of the value can be considered as wholly satisfied. It should be said that Eq.(43) does not working well in case with the Sun. The Sun surface rotates with period $T \approx 25 \div 30$ days. At this velocity of rotation, the magnetic field on the Sun pole calculated accordingly to Eq.(43) must be about $1 \mathrm{kOe}$. The dipole field of Sun according to experts estimation is approximately 20 times lower. There can be several reasons for that.

\section{The angular velocity of the apsidal rotation in binary stars}

\subsection{The apsidal rotation of close binary stars}

The apsidal rotation (or periastron rotation) of close binary stars is a result of their non-Keplerian movement which originates from the non-spherical form of stars. This non-sphericity has been produced by rotation of stars around their axes or by their mutual tidal effect. The second effect is usually smaller and can be neglected. The first and basic theory of this effect was developed by A.Clairault at the beginning of the XVIII century. Now this effect was measured for approximately 50 double stars. According to Clairault's theory the velocity of periastron rotation must be approximately 100 times faster if matter is uniformly distributed inside a star. Reversely, it would be absent if all star mass is concentrated in the star center. To reach an agreement between the measurement data and calculations, it is necessary to assume that the density of substance grows in direction to the center of a star and here it runs up to a value which is hundreds times greater than mean density of a star. Just the same mass concentration of the stellar substance is supposed by all standard theories of a star interior. It has been usually considered as a proof of astrophysical models. But it can be considered as a qualitative argument. To obtain a quantitative agreement between theory and measurements, it is necessary to fit parameters of the stellar substance distribution in each case separately.

Let us consider this problem with taking into account the gravity induced electric polarization of plasma in a star. As it was shown above, one half of full mass of a star is concentrated in its plasma core at a permanent density. Therefor, the effect of periastron rotation of close binary stars must be reviewed with the account of a change of forms of these star cores. 
According to [10],[9] the ratio of the angular velocity $\omega$ of rotation of periastron which is produced by the rotation of a star around its axis with the angular velocity $\Omega$ is

$$
\frac{\omega}{\Omega}=\frac{3}{2} \frac{\left(I_{A}-I_{C}\right)}{M a^{2}}
$$

where $I_{A}$ and $I_{C}$ are the moments of inertia relatively to principal axes of the ellipsoid. Their difference is

$$
I_{A}-I_{C}=\frac{M}{5}\left(a^{2}-c^{2}\right)
$$

where $a$ and $c$ are the equatorial and polar radii of the star.

Thus we have

$$
\frac{\omega}{\Omega} \approx \frac{3}{10} \frac{\left(a^{2}-c^{2}\right)}{a^{2}} .
$$

\subsection{The equilibrium form of the core of a rotating star}

In the absence of rotation the equilibrium equation of plasma inside star core Eq.(I-41) is

$$
\gamma \mathbf{g}_{G}+\rho_{G} \mathbf{E}_{G}=0
$$

where $\gamma, \mathbf{g}_{G}, \rho_{G}$ and $\mathbf{E}_{G}$ are the substance density the acceleration of gravitation, gravity-induced density of charge and intensity of gravity-induced electric field ( $\operatorname{div} \mathbf{g}_{G}=4 \pi G \gamma$, div $\mathbf{E}_{G}=4 \pi \rho_{G}$ and $\rho_{G}=\sqrt{G} \gamma$ ).

One can suppose, that at rotation, under action of a rotational acceleration $\mathbf{g}_{\Omega}$, an additional electric charge with density $\rho_{\Omega}$ and electric field $\mathbf{E}_{\Omega}$ can exist, and the equilibrium equation obtains the form:

$$
\left(\gamma_{G}+\gamma_{\Omega}\right)\left(\mathbf{g}_{G}+\mathbf{g}_{\Omega}\right)=\left(\rho_{G}+\rho_{\Omega}\right)\left(\mathbf{E}_{G}+\mathbf{E}_{\Omega}\right)
$$

where

$$
\operatorname{div}\left(\mathbf{E}_{G}+\mathbf{E}_{\Omega}\right)=4 \pi\left(\rho_{G}+\rho_{\Omega}\right)
$$

or

$$
\operatorname{div} \mathbf{E}_{\Omega}=4 \pi \rho_{\Omega} .
$$

We can look for a solution for electric potential in the form

$$
\varphi=C_{\Omega} r^{2}\left(3 \cos ^{2} \theta-1\right)
$$

or in Cartesian coordinates

$$
\varphi=C_{\Omega}\left(3 z^{2}-x^{2}-y^{2}-z^{2}\right)
$$

where $C_{\Omega}$ is a constant.

Thus

$$
E_{x}=2 C_{\Omega} x, E_{y}=2 C_{\Omega} y, E_{z}=-4 C_{\Omega} z
$$

and

$$
\operatorname{div} \mathbf{E}_{\Omega}=0
$$

and we obtain important equations:

$$
\begin{gathered}
\rho_{\Omega}=0 ; \\
\gamma g_{\Omega}=\rho \mathbf{E}_{\Omega} .
\end{gathered}
$$

Since centrifugal force must be contra-balanced by electric force

$$
\gamma 2 \Omega^{2} x=\rho 2 C_{\Omega} x
$$

and

$$
C_{\Omega}=\frac{\gamma \Omega^{2}}{\rho}=\frac{\Omega^{2}}{\sqrt{G}}
$$

The potential of a positive uniform charged ball is 


$$
\varphi(r)=\frac{Q}{R}\left(\frac{3}{2}-\frac{r^{2}}{2 R^{2}}\right)
$$

The negative charge on the surface of a sphere induces inside the sphere the potential

$$
\varphi(R)=-\frac{Q}{R}
$$

where according to Eq.(47) $Q=\sqrt{G} M$, and $M$ is the mass of the star.

Thus the total potential inside the considered star is

$$
\varphi_{\Sigma}=\frac{\sqrt{G} M}{2 R}\left(1-\frac{r^{2}}{R^{2}}\right)+\frac{\Omega^{2}}{\sqrt{G}} r^{2}\left(3 \cos ^{2} \theta-1\right)
$$

Since the electric potential must be equal to zero on the surface of the star, at $r=a$ and $r=c$

$$
\varphi_{\Sigma}=0
$$

and we obtain the equation which describes the equilibrium form of the core of a rotating $\operatorname{star}\left(\right.$ at $\left.\frac{a^{2}-c^{2}}{a^{2}} \ll 1\right)$

$$
\frac{a^{2}-c^{2}}{a^{2}} \approx \frac{9}{2 \pi} \frac{\Omega^{2}}{G \gamma}
$$

\subsection{The angular velocity of the apsidal rotation}

Taking into account of Eq.(63) we have

$$
\frac{\omega}{\Omega} \approx \frac{27}{20 \pi} \frac{\Omega^{2}}{G \gamma}
$$

If both stars of a close pair induce a rotation of periastron, this equation transforms to

$$
\frac{\omega}{\Omega} \approx \frac{27}{20 \pi} \frac{\Omega^{2}}{G}\left(\frac{1}{\gamma_{1}}+\frac{1}{\gamma_{2}}\right),
$$

where $\gamma_{1}$ and $\gamma_{2}$ are densities of star cores.

The equilibrium density of star cores is known (Eq.(I-22)):

$$
\gamma=\frac{16}{9 \pi^{2}} \frac{A}{Z} m_{p} \frac{Z^{3}}{a_{B}^{3}} .
$$

If we introduce the period of ellipsoidal rotation $P=\frac{2 \pi}{\Omega}$ and the period of the rotation of periastron $U=\frac{2 \pi}{\omega}$, we obtain from Eq.(64)

$$
\frac{\mathcal{P}}{\mathcal{U}}\left(\frac{\mathcal{P}}{\mathcal{T}}\right)^{2} \approx \sum_{1}^{2} \xi_{i}
$$

where

$$
\begin{gathered}
\mathcal{T}=\sqrt{\frac{243 \pi^{3}}{80}} \tau_{0} \approx 10 \tau_{0}, \\
\tau_{0}=\sqrt{\frac{a_{B}^{3}}{G m_{p}}} \approx 7.7 \cdot 10^{2} \mathrm{sec}
\end{gathered}
$$

and

$$
\xi_{i}=\frac{Z_{i}}{A_{i}\left(Z_{i}+1\right)^{3}}
$$

\subsection{The comparison of the calculated angular velocity of the periastron rotation with observa- tions}

Because the substance density (Eq.(66)) is depending approximately on the second power of the nuclear charge, the periastron movement of stars consisting of heavy elements will fall out from the observation as it is very slow. Practically the obtained equation (67) shows that it is possible to observe the periastron rotation of a star consisting of light elements only.

The value $\xi=Z /\left[A Z^{3}\right]$ is equal to $1 / 8$ for hydrogen, 0.0625 for deuterium, $1.85 \cdot 10^{-2}$ for helium. The resulting value of the periastron rotation of double stars will be the sum of separate stars rotation. The possible combinations of a couple and their value of $\sum_{1}^{2} \xi_{i}$ for stars consisting of light elements is shown in Table 2. 


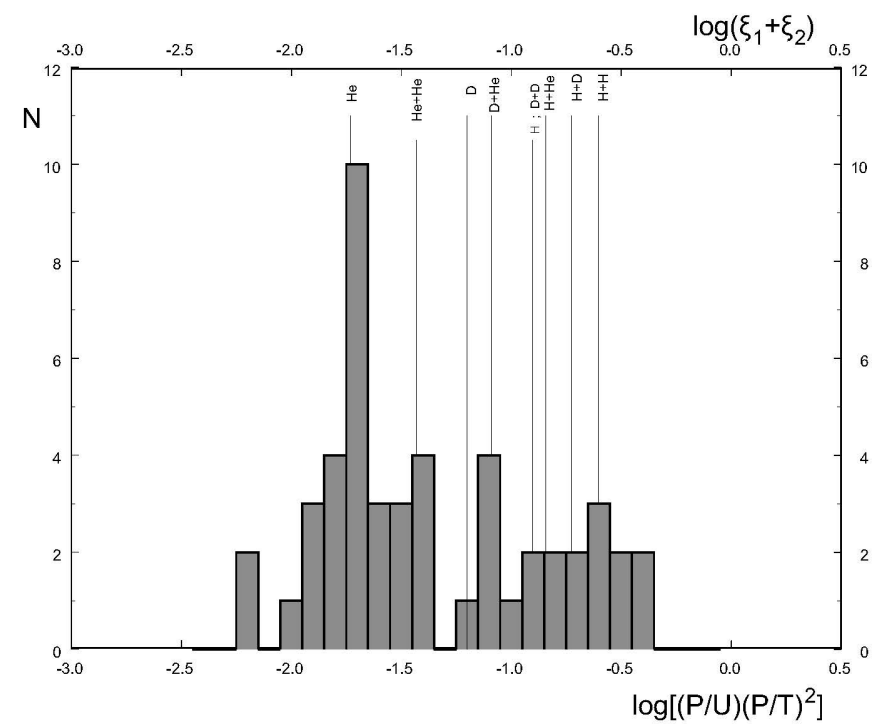

Figure 7. The distribution of close binary stars [3] on value of $(\mathcal{P} / \mathcal{U})(\mathcal{P} / \mathcal{T})^{2}$. Lines show parameters $\sum_{1}^{2} \xi_{i}$ for different light atoms in according with 70 .

\begin{tabular}{||c|c|c||}
\hline \hline $\begin{array}{c}\text { star1 } \\
\text { composed of }\end{array}$ & $\begin{array}{c}\text { star2 } \\
\text { composed of }\end{array}$ & $\xi_{1}+\xi_{2}$ \\
\hline $\mathrm{H}$ & $\mathrm{H}$ & .25 \\
$\mathrm{H}$ & $\mathrm{D}$ & 0.1875 \\
$\mathrm{H}$ & $\mathrm{He}$ & 0.143 \\
$\mathrm{H}$ & $\mathrm{hn}$ & 0.125 \\
$\mathrm{D}$ & $\mathrm{D}$ & 0.125 \\
$\mathrm{D}$ & $\mathrm{He}$ & 0.0815 \\
$\mathrm{D}$ & $\mathrm{hn}$ & 0.0625 \\
$\mathrm{He}$ & $\mathrm{He}$ & 0.037 \\
$\mathrm{He}$ & $\mathrm{hn}$ & 0.0185 \\
\hline \hline
\end{tabular}

Table 2

The possible combinations of a couple and their value of $\sum_{1}^{2} \xi_{i}$ for stars consisting of light elements.

The "hn" notation in Table 2 indicates that the second component of the couple consists of heavy elements or it is a dwarf.

The results of measuring of main parameters for close binary stars are gathered in [3]. For reader convenience, the data of these measurement is applied in the Table in Appendix. One can compare our calculations with data of these measurements. The distribution of close binary stars on value of $(\mathcal{P} / \mathcal{U})(\mathcal{P} / \mathcal{T})^{2}$ is shown in Fig.7 on logarithmic scale. The lines mark the values of parameters $\sum_{1}^{2} \xi_{i}$ for different light atoms in accordance with 70 . It can be seen that calculated values the periastron rotation for stars composed by light elements which is summarized in Table 2 are in good agreement with separate peaks of measured data. It confirms that our approach to interpretation of this effect is adequate to produce a satisfactory accuracy of estimations.

\section{The solar seismical oscillations}

\subsection{The spectrum of solar seismic oscillations}

The measurements [11] show that the Sun surface is subjected to a seismic vibration. The most intensive oscillations have the period about five minutes and the wave length about $10^{4} \mathrm{~km}$ or about hundredth part of the Sun radius. Their spectrum obtained by BISON collaboration is shown in Fig.8.

It is supposed, that these oscillations are a superposition of a big number of different modes of resonant acoustic vibrations, and that acoustic waves propagate in different trajectories in the interior of the Sun and they have multiple reflection from surface. With these reflections trajectories of same waves can be closed and as a result standing waves are forming.

Specific features of spherical body oscillations are described by the expansion in series on spherical functions. These oscillations can have a different number of wave lengths on the radius of a sphere $(n)$ and a different number of wave lengths on its surface which is determined by the $l$-th spherical harmonic. It is accepted to describe the sunny surface oscillation spectrum as the expansion in series [12]:

$$
\nu_{n l m} \simeq \Delta \nu_{0}\left(n+\frac{l}{2}+\epsilon_{0}\right)-l(l+1) D_{0}+m \Delta \nu_{r o t} .
$$



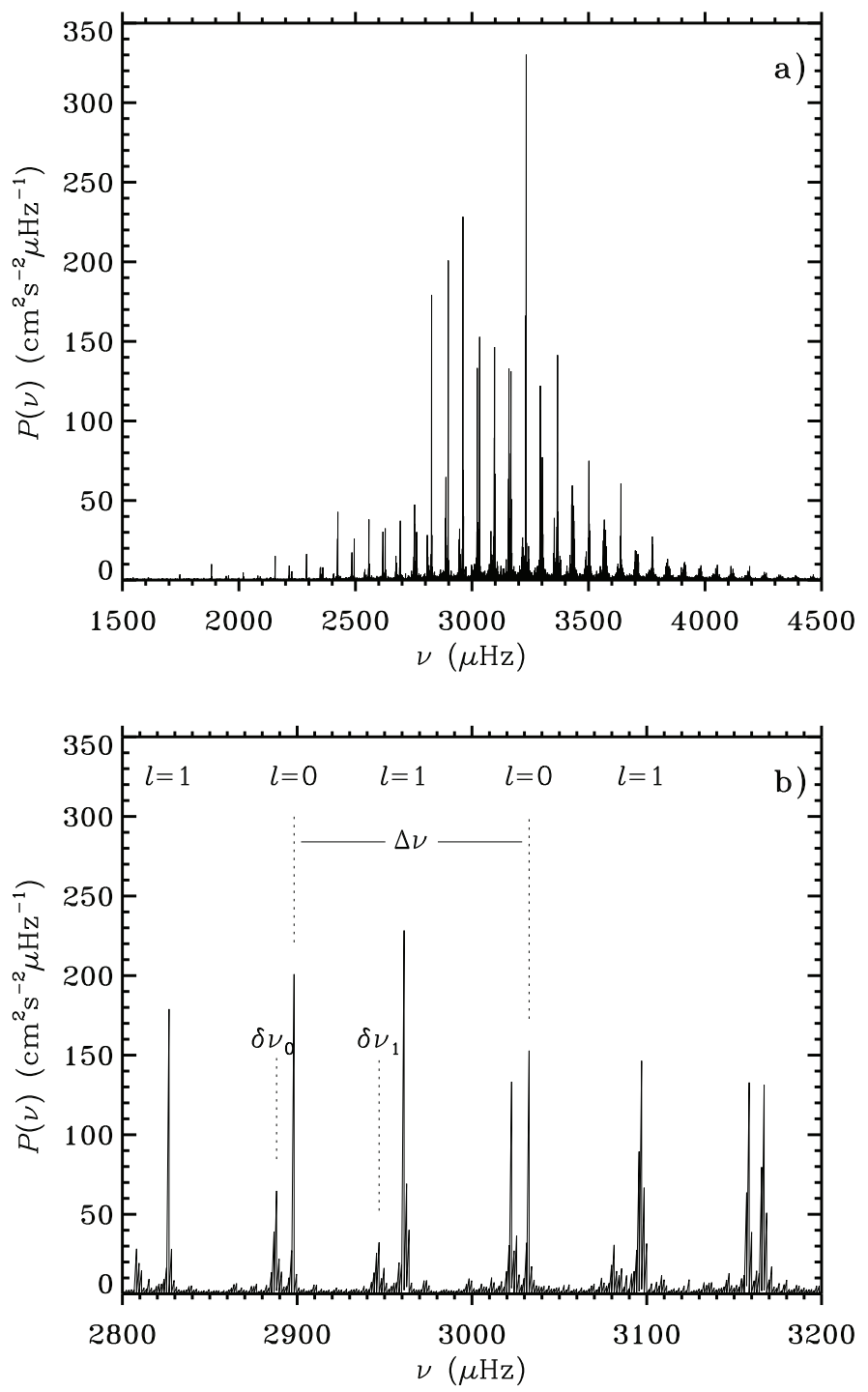

Figure 8. $(a)$ The power spectrum of solar oscillation obtained by means of Doppler velocity measurement in light integrated over the solar disk. The data were obtained from the BISON network [11]. (b) An expanded view of a part of frequency range. 

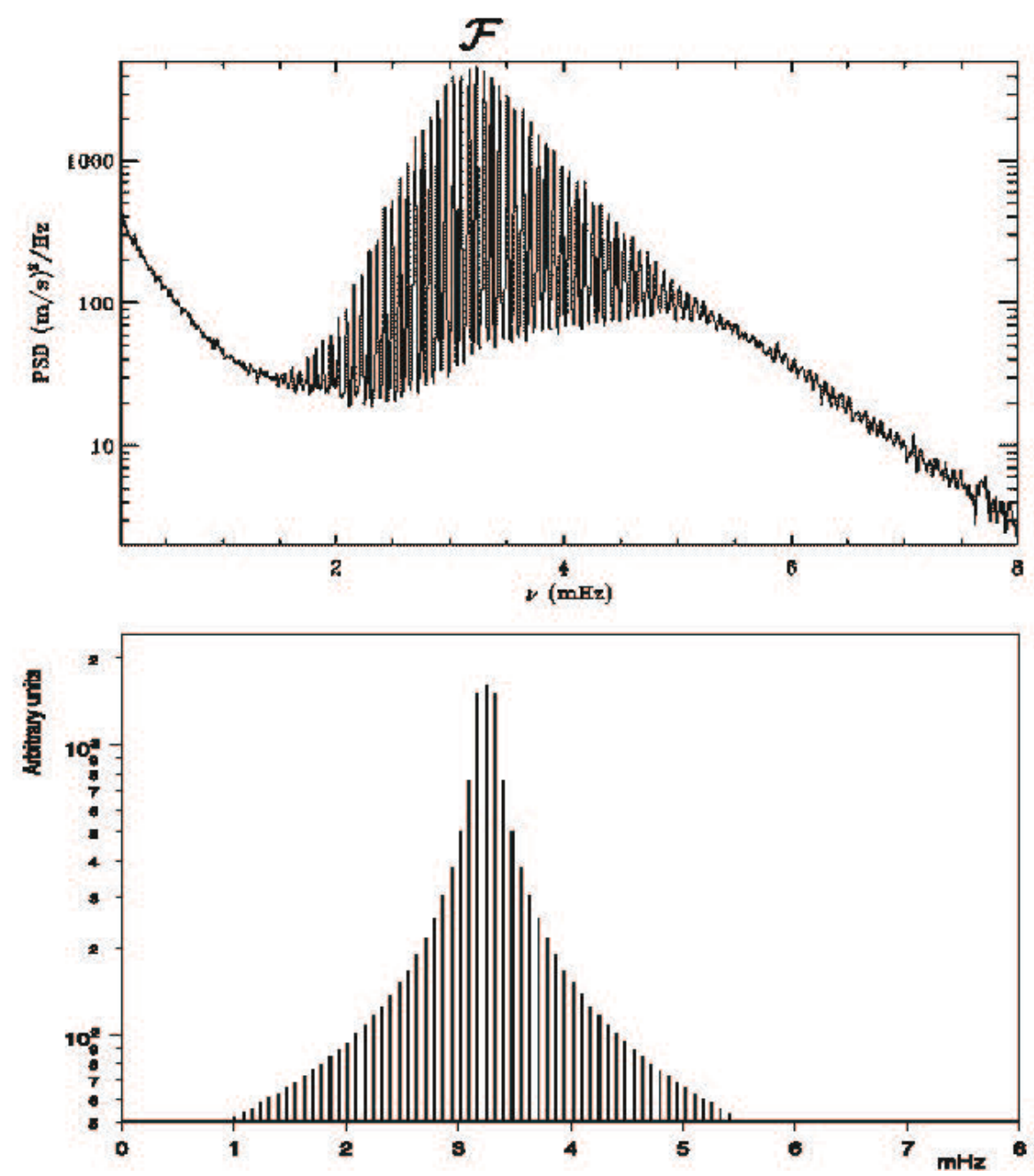

Figure 9. (a) The measured power spectrum of solar oscillation. The data were obtained from the SOHO/GOLF measurement [13]. (b) The calculated spectrum described by Eq.(96) at $\langle Z\rangle=3.4$ and $A / Z=5$.

Where the last item is describing the effect of the Sun rotation and is small. The main contribution is given by the first item which creates a large splitting in the spectrum (Fig.8)

$$
\triangle \nu=\nu_{n+1, l}-\nu_{n, l}
$$

The small splitting of spectrum (Fig.8) depends on the difference

$$
\delta \nu_{l}=\nu_{n, l}-\nu_{n-1, l+2} \approx(4 l+6) D_{0} .
$$

A satisfactory agreement of these estimations and measurement data can be obtained at [12]

$$
\Delta \nu_{0}=120 \mu \mathrm{Hz}, \epsilon_{0}=1.2, D_{0}=1.5 \mu \mathrm{Hz}, \Delta \nu_{\text {rot }}=1 \mu \mathrm{Hz} .
$$

To obtain these values of parameters $\Delta \nu_{0}, \epsilon_{0} D_{0}$ from theoretical models is not possible. There are a lot of qualitative and quantitative assumptions used at a model construction and a direct calculation of spectral frequencies transforms into a unresolved complicated problem.

Thus, the current interpretation of the measuring spectrum by the spherical harmonic analysis does not make it clear. It gives no hint to an answer to the question: why oscillations close to hundredth harmonics are really excited and there are no waves near fundamental harmonic?

The measured spectra have a very high resolution (see Fig.(8)). It means that an oscillating system has high quality. At this condition, the system must have oscillation on a fundamental frequency. Some peculiar mechanism must exist to force a system to oscillate on a high harmonic. The current explanation does not clarify it.

It is important, that now the solar oscillations are measured by means of two different methods. The solar oscillation spectra which was obtained on program "BISON", is shown on Fig.(8)). It has a very high resolution, but (accordingly to the Liouville's theorem) it was obtained with some loss of luminosity, and as a result not all lines are well statistically worked.

Another spectrum was obtained in the program "SOHO/GOLF". Conversely, it is not characterized by high resolution, instead it gives information about general character of the solar oscillation spectrum (Fig.9)).

The existence of this spectrum requires to change the view at all problems of solar oscillations. The theoretical explanation of this spectrum must give answers at least to four questions: 
1.Why does the whole spectrum consist from a large number of equidistant spectral lines?

2. Why does the central frequency of this spectrum $\mathcal{F}$ is approximately equal to $\approx 3.23 \mathrm{mHz}$ ?

3. Why does this spectrum splitting $f$ is approximately equal to $67.5 \mu \mathrm{Hz}$ ?

4. Why does the intensity of spectral lines decrease from the central line to the periphery?

The answers to these questions can be obtained if we take into account electric polarization of a solar core.

The description of measured spectra by means of spherical analysis does not make clear of the physical meaning of this procedure. The reason of difficulties lies in attempt to consider the oscillations of a Sun as a whole. At existing dividing of a star into core and atmosphere, it is easy to understand that the core oscillation must form a measured spectrum. The fundamental mode of this oscillation must be determined by its spherical mode when the Sun radius oscillates without changing of the spherical form of the core. It gives a most low-lying mode with frequency:

$$
\Omega_{s} \approx \frac{c_{s}}{\mathbb{R}_{\star}}
$$

where $c_{s}$ is sound velocity in the core.

It is not difficult to obtain the numerical estimation of this frequency by order of magnitude. Supposing that the sound velocity in dense matter is $10^{7} \mathrm{~cm} / \mathrm{c}$ and radius is close to $\frac{1}{10}$ of external radius of a star, i.e. about $10^{10} \mathrm{~cm}$, one can obtain as a result

$$
F=\frac{\Omega_{s}}{2 \pi} \approx 10^{-3} H z
$$

It gives possibility to conclude that this estimation is in agreement with measured frequencies. Let us consider this mechanism in more detail.

\subsection{The sound speed in hot plasma}

The pressure of high temperature plasma is a sum of the plasma pressure (ideal gas pressure) and the pressure of black radiation:

$$
P=n_{e} k T+\frac{\pi^{2}}{45 \hbar^{3} c^{3}}(k T)^{4} .
$$

and its entropy is

$$
S=\frac{1}{\frac{A}{Z} m_{p}} \ln \frac{(k T)^{3 / 2}}{n_{e}}+\frac{4 \pi^{2}}{45 \hbar^{3} c^{3} n_{e}}(k T)^{3},
$$

The sound speed $c_{s}$ can be expressed by Jacobian [2]:

$$
c_{s}^{2}=\frac{D(P, S)}{D(\rho, S)}=\frac{\left(\frac{D(P, S)}{D\left(n_{e}, T\right)}\right)}{\left(\frac{D(\rho, S)}{D\left(n_{e}, T\right)}\right)}
$$

or

$$
c_{s}=\left\{\frac{5}{9} \frac{k T}{A / Z m_{p}}\left[1+\frac{2\left(\frac{4 \pi^{2}}{45 \hbar^{3} c^{3}}\right)^{2}(k T)^{6}}{5 n_{e}\left[n_{e}+\frac{8 \pi^{2}}{45 \hbar^{3} c^{3}}(k T)^{3}\right]}\right]\right\}^{1 / 2}
$$

For $T=\mathbb{T}_{\star}$ and $n_{e}=n_{\star}$ we have:

$$
\frac{4 \pi^{2}\left(k \mathbb{T}_{\star}\right)^{3}}{45 \hbar^{3} c^{3} n_{\star}}=\approx 0.18
$$

Finally we obtain:

$$
c_{s}=\left\{\frac{5}{9} \frac{\mathbb{T}_{\star}}{(A / Z) m_{p}}[1.01]\right)^{1 / 2} \approx 3.1410^{7}\left(\frac{Z}{A / Z}\right)^{1 / 2} \mathrm{~cm} / \mathrm{s}
$$

\subsection{The basic elastic oscillation of a spherical core}

Star cores consist of dense high temperature plasma which is a compressible matter. The basic mode of elastic vibrations of a spherical core is related with its radius oscillation. For the description of this type of oscillation, the potential $\phi$ of displacement velocities $v_{r}=\frac{\partial \psi}{\partial r}$ can be introduced and the motion equation can be reduced to the wave equation expressed through $\phi[2]$ :

$$
c_{s}^{2} \Delta \phi=\ddot{\phi}
$$

and a spherical derivative for periodical in time oscillations $\left(\sim e^{-i \Omega_{s} t}\right)$ is:

$$
\Delta \phi=\frac{1}{r^{2}} \frac{\partial}{\partial r}\left(r^{2} \frac{\partial \phi}{\partial r}\right)=-\frac{\Omega_{s}^{2}}{c_{s}^{2}} \phi
$$


It has the finite solution for the full core volume including its center

$$
\phi=\frac{A}{r} \sin \frac{\Omega_{s} r}{c_{s}}
$$

where $A$ is a constant. For small oscillations, when displacements on the surface $u_{R}$ are small $\left(u_{R} / R=v_{R} / \Omega_{s} R \rightarrow 0\right)$ we obtain the equation:

$$
\operatorname{tg} \frac{\Omega_{s} \mathbb{R}}{c_{s}}=\frac{\Omega_{s} \mathbb{R}}{c_{s}}
$$

which has the solution:

$$
\frac{\Omega_{s} \mathbb{R}}{c_{s}} \approx 4.49
$$

Taking into account Eq.(82)), the main frequency of the core radial elastic oscillation is

$$
\Omega_{s}=4.49\left\{1.4\left[\frac{G m_{p}}{r_{B}^{3}}\right] \frac{A}{Z}(Z+1)^{3}\right\}^{1 / 2} .
$$

It can be seen that this frequency depends on $Z$ and $A / Z$ only. Some values of frequencies of radial sound oscillations $\mathcal{F}=\Omega_{s} / 2 \pi$ calculated from this equation for selected $A / Z$ and $Z$ are shown in third column of Table 3.

\begin{tabular}{||c|c|c||c|c||}
\hline \hline $\mathrm{Z}$ & $\mathrm{A} / \mathrm{Z}$ & $\begin{array}{c}\mathcal{F}, m \mathrm{mz} \\
\left(\begin{array}{c}\text { calculated } \\
(88))\end{array}\right.\end{array}$ & star & $\mathcal{F}, m \mathrm{~Hz}$ \\
\hline 1 & 1 & 0.23 & $\xi$ Hydrae & $\sim 0.1$ \\
\hline 1 & 2 & 0.32 & $\nu$ Indus & 0.3 \\
\hline 2 & 2 & 0.9 & $\eta$ Bootis & 0.85 \\
\hline 2 & 3 & 1.12 & The Procion $($ A $\alpha$ CMi $)$ & 1.04 \\
\hline 3 & 4 & 2.38 & $\beta$ Hydrae & 1.08 \\
\hline \hline 3 & 5 & 2.66 & $\alpha$ Cen $A$ & 2.37 \\
\hline 3.4 & 5 & $\mathbf{3 . 2 4}$ & & $\mathbf{3 . 2 3}$ \\
\hline 4 & 5 & 4.1 & & \\
\hline
\end{tabular}

Table 3. Calculated and measured frequencies of seismic oscillations of stars.

The star mass spectrum (Fig.(I-1)) shows that the ratio $A / Z$ must be $\approx 5$ for the Sum. It is in accordance with the calculated frequency of solar core oscillations if the averaged charge of nuclei $Z \approx 3.4$. It is not a confusing conclusion, because the plasma electron gas prevents the decay of $\beta$-active nuclei (see Sec.(III-1). This mechanism can probably to stabilize neutron-excess nuclei.

\subsection{The low frequency oscillation of the density of a neutral plasma}

Hot plasma has the density $n_{\star}$ at its equilibrium state. The local deviations from this state induce processes of density oscillation since plasma tends to return to its steady-state density. If we consider small periodic oscillations of core radius

$$
R=\mathbb{R}+u_{R} \cdot \sin \omega_{n_{\star}} t
$$

where a radial displacement of plasma particles is small $\left(u_{R} \ll \mathbb{R}\right)$, the oscillation process of plasma density can be described by the equation

$$
\frac{d \mathcal{E}}{d R}=\mathbb{M} \ddot{R}
$$

Taking into account

$$
\frac{d \mathcal{E}}{d R}=\frac{d \mathcal{E}_{\text {plasma }}}{d n_{e}} \frac{d n_{e}}{d R}
$$

and

$$
\frac{3}{8} \pi^{3 / 2} \mathbb{N}_{e} \frac{e^{3} a_{0}^{3 / 2}}{(k \mathbb{T})^{1 / 2}} \frac{n_{\star}}{\mathbb{R}^{2}}=\mathbb{M} \omega_{n_{\star}}^{2}
$$

From this we obtain

$$
\omega_{n_{\star}}^{2}=\frac{3}{\pi^{1 / 2}} k \mathbb{T}\left(\frac{e^{2}}{a_{B} k \mathbb{T}}\right)^{3 / 2} \frac{Z^{3}}{\mathbb{R}^{2} A / Z m_{p}}
$$


and finally

$$
\omega_{n_{\star}}=\left\{\frac{2^{8}}{3^{5}} \frac{\pi^{1 / 2}}{10^{1 / 2}} \alpha^{3 / 2}\left[\frac{G m_{p}}{a_{B}^{3}}\right] \frac{A}{Z} Z^{4.5}\right\}^{1 / 2},
$$

where $\alpha=\frac{e^{2}}{\hbar c}$ is the fine structure constant. These low frequency oscillations of neutral plasma density are similar to phonons in solid bodies. At that oscillations with multiple frequencies $k \omega_{n_{\star}}$ can exist. Their power is proportional to $1 / \kappa$, as the occupancy these levels in energy spectrum must be reversely proportional to their energy $k \hbar \omega_{n_{\star}}$. As result, low frequency oscillations of plasma density constitute set of vibrations

$$
\sum_{\kappa=1} \frac{1}{\kappa} \sin \left(\kappa \omega_{n_{\star}} t\right)
$$

\subsection{The spectrum of solar core oscillations}

The set of the low frequency oscillations with $\omega_{\eta}$ can be induced by sound oscillations with $\Omega_{s}$. At that, displacements obtain the spectrum:

$$
u_{R} \sim \sin \Omega_{s} t \cdot \sum_{\kappa=0} \frac{1}{\kappa} \sin \kappa \omega_{n_{\star}} t \cdot \sim \xi \sin \Omega_{s} t+\sum_{\kappa=1} \frac{1}{\kappa} \sin \left(\Omega_{s} \pm \kappa \omega_{n_{\star}}\right) t,
$$

where $\xi$ is a coefficient $\approx 1$.

This spectrum is shown in Fig.(9).

The central frequency of experimentally measured distribution of solar oscillations is approximately equal to (Fig.(8))

$$
\mathcal{F}_{\odot} \approx 3.23 \mathrm{mHz}
$$

and the experimentally measured frequency splitting in this spectrum is approximately equal to

$$
f_{\odot} \approx 68 \mu H z
$$

A good agreement of the calculated frequencies of basic modes of oscillations (from Eq.(88) and Eq.(47)) with measurement can be obtained at $Z=3.4$ and $A / Z=5$ :

$$
\mathcal{F}_{z=3.4 ; \frac{A}{Z}=5}=\frac{\Omega_{s}}{2 \pi}=3.24 \mathrm{mHz} ; f_{z=3.4 ; \frac{A}{Z}=5}=\frac{\omega_{n_{\star}}}{2 \pi}=68.1 \mu \mathrm{Hz} .
$$

\section{REFERENCES}

[1] Vasiliev B.V. and Luboshits V.L.: Physics-Uspekhi, 37, 345, (1994)

[2] Landau L.D. and Lifshits E.M.: Statistical Physics, 1, 3rd edition, Oxford:Pergamon, (1980)

[3] Khaliullin K.F.: Dissertation, Sternberg Astronomical Institute, Moscow, (Russian)(2004) (see Table in Appendix)

[4] Blackett P.M.S.: Nature, 159, 658, (1947)

[5] Sirag S.-P.: Nature, 275, 535, (1979)

[6] Beskin V.S., Gurevich A.V., Istomin Ya.N.: Physics of the Pulsar Magnetosphere (Cambridge University Press) (1993)

[7] Thorsett S.E. and Chakrabarty D.: E-preprint: astro-ph/9803260, (1998)

[8] I.I.Romanyuk at al. Magnetic Fields of Chemically Peculiar and Related Stars, Proceedings of the International Conference (Nizhnij Arkhyz, Special Astrophysical Observatory of Russian Academy of Sciences, September 24-27, 1999), eds: Yu. V. Glagolevskij and I.I. Romanyuk, Moscow,2000, pp. 18-50.

[9] Russel H.N.: Monthly Notices of the RAS 88, 642, (1928)

[10] Chandrasekhar S.: Monthly Notices of the RAS 93, 449, (1933)

[11] Elsworth, Y. at al. - In Proc. $\mathcal{G} O N G^{\prime} 94$ Helio- and Astero-seismology from Earth and Space, eds. Ulrich,R.K., Rhodes Jr,E.J. and Däppen,W., Asrtonomical Society of the Pasific Conference Series, vol.76, San Fransisco,76, 51-54.

[12] Christensen-Dalsgaard, J.: Stellar oscillation, Institut for Fysik og Astronomi, Aarhus Universitet, Denmark, (2003)

[13] Solar Physics, 175/2,

(http://sohowww.nascom.nasa.gov/gallery/Helioseismology) 